\title{
The Transmission Property of the Discrete Heisenberg Ferromagnetic Spin Chain
}

\author{
Qing Ding* and Wei Lin \\ Inst. of Math. and Key Lab. of Math. for Nonlinear Sciences \\ Fudan University, Shanghai 200433, P.R. China
}

\begin{abstract}
We present a mechanism for displaying the transmission property of the discrete Heisenberg ferromagnetic spin chain (DHF) via a geometric approach. By the aid of a discrete nonlinear Schrödinger-like equation which is the discrete gauge equivalent to the DHF, we show that the determination of transmitting coefficients in the transmission problem is always bistable. Thus a definite algorithm and general stochastic algorithms are presented. A new invariant periodic phenomenon of the non-transmitting behavior for the DHF, with a large probability, is revealed by an adoption of various stochastic algorithms.
\end{abstract}

PACS numbers: 02.40.Ky; 05.45.Mt; 07.55.Db

\section{$\S 1$ Introduction}

There is current interest in displaying the properties of one-dimensional magnetic models. The one-dimensional classical continuum Heisenberg models with different magnetic interactions have been settled as one of the interesting and attractive class of nonlinear dynamical equations exhibiting the complete integrability on many occasions ([1]-[7]). However, though the investigation of nonlinear spin chain systems is quite fascinating, few has been known in the case of more realistic physical lattice spin chains so far, especially, for the following discrete (isotropic) Heisenberg ferromagnetic spin chain with nearest neighbor exchange interaction (DHF),

$$
\dot{\mathbf{S}}_{n}=\mathbf{S}_{n} \times\left(\mathbf{S}_{n+1}+\mathbf{S}_{n-1}\right),
$$

where $\mathbf{S}_{n}=\left(s_{n}^{1}, s_{n}^{2}, s_{n}^{3}\right) \in \mathbf{R}^{3}$ with $\left(s_{n}^{1}\right)^{2}+\left(s_{n}^{2}\right)^{2}+\left(s_{n}^{3}\right)^{2}=1$ and the dot stands for the time derivative. In fact Eq.(1) comes from the Hamiltonian formalism: $\dot{\mathbf{S}}_{n}=\left\{\mathbf{S}_{n}, H\right\}$ with the Hamiltonian function $H=\sum_{j=-\infty}^{+\infty} \mathbf{S}_{j} \cdot \mathbf{S}_{j+1}$, where $\cdot$ denotes the inner product of vectors in $\mathbf{R}^{3}$, and the Poisson bracket $\left\{s_{j}^{a}, s_{k}^{b}\right\}=\delta_{j k} \sum_{c=1}^{3} \varepsilon_{a b c} s_{j}^{c}$, where $\delta_{j k}$ is Kronecker's

\footnotetext{
*qding@fudan.edu.cn
} 
symbol and $\varepsilon_{a b c}$ is the 3-dimensional totally antisymmetric symbol. Physically, one would say the model (1) describes a system of classical spins and subjects to homogenous nearestneighbor Heisenberg interaction. The standard continuous limit procedure performed on Eq.(1) leads to the integrable Heisenberg ferromagnetic model: $\mathbf{S}_{t}=\mathbf{S} \times \mathbf{S}_{x x}$, which is an important equation on condensed matter physics (see, for example, [8]). Eq.(11) is quite well known and there is little need to give here a detailed enumeration. It should be also emphasized that Eq.(11) is widely believed to be not a completely integrable equation. To our best knowledge, there is no effective method in study of the dynamical behaviors of Eq.(11) in literature.

On the other hand, the geometric concept of gauge equivalence [9, 10] between integrable equations, which provides a useful tool in displaying solitonic dynamics, has been generalized to nonintegrable case in [11, 12]. In [12] it is shown that the discrete nonlinear (nonintegrable) Schrödinger equation (AL-DNLS) is the discrete gauge equivalent to a nonintegrable discrete Heisenberg model and the transmission properties of the AL-DNLS equation ([13]-[18]) are completely preserved under the action of discrete gauge transformations. In this paper, we would apply this geometric idea to transform the DHF (11) into a discrete nonlinear Schrödinger-like equation and utilize the nonlinear Schrödinger-like equation to study the transmission problem of the DHF (1) from mathematical point of view. We hope that this study will be helpful to reveal deeper quantum chaotic properties of the DHF (11) and useful in physical applications.

This article is organized as follows. In section 2 we deduce a discrete nonlinear Schödinger-type equation which is the discrete gauge equivalent to the DHF in the category of the (discrete) Yang-Mills theory. In section 3 we display the transmission property for the DHF (11) with the aid of the discrete nonlinear Schödinger-type equation and an approximate linear stability analysis for the stationary version of the discrete nonlinear Schödinger-type equation is given for supporting the discussion of the transmission expositions. Finally, in section 4, we close the paper with some conclusions and remarks.

\section{$\S 2$ Gauge equivalence}

Following the concept of discrete connection and associated discrete curvature introduced in [12], we would express Eq.(11) geometrically as a discrete nonlinear equation with prescribed discrete curvature representation. We first convert Eq.(1) into its matrix version:

$$
\dot{S}_{n}=-i \frac{\left[S_{n}, S_{n+1}\right]}{2}-i \frac{\left[S_{n}, S_{n-1}\right]}{2},
$$

where $S_{n}=\left(\begin{array}{cc}s_{n}^{1} & s_{n}^{2}-i s_{n}^{3} \\ s_{n}^{2}+i s_{n}^{3} & -s_{n}^{1}\end{array}\right)$. Then we define a discrete connection $\left\{A_{n}\right\}$ by

$$
A_{n}=\left(L_{n}, M_{n}\right),
$$


where $L_{n}=\frac{z+z^{-1}}{2} I+\frac{z-z^{-1}}{2} S_{n}, M_{n}=i\left(1-\frac{z^{2}+z^{-2}}{2}\right) \frac{S_{n}+S_{n-1}}{2}-i \frac{z^{2}-z^{-2}}{2} \frac{I+S_{n-1} S_{n}}{2}$ with $I$ denoting the $2 \times 2$ identity matrix and $z$ being a free spectral parameter. It is a direct and lengthy computation, in the similar way displayed in the appendix of [12], that the corresponding discrete curvature $\left\{F_{n}^{A}\right\}$ is given by $F_{n}^{A}:=\dot{L}_{n}-M_{n+1} L_{n}+$ $L_{n} M_{n}=\frac{z-z^{-1}}{2}\left(\dot{S}_{n}+i \frac{\left[S_{n}, S_{n-1}\right]}{2}+i \frac{\left[S_{n}, S_{n+1}\right]}{2}\right)+i \frac{-z^{3}+z+z^{-1}-z^{-3}}{4}\left(\mathbf{S}_{n} \cdot \mathbf{S}_{n-1}-\mathbf{S}_{n} \cdot \mathbf{S}_{n+1}\right) S_{n}+$ $i \frac{-z^{3}+z-z^{-1}+z^{-3}}{4}\left(\mathbf{S}_{n} \cdot \mathbf{S}_{n-1}-\mathbf{S}_{n} \cdot \mathbf{S}_{n+1}\right) I$. Here we have used the identity: $S_{n+1} S_{n}=$ $-S_{n} S_{n+1}+\left(\mathbf{S}_{n} \cdot \mathbf{S}_{n+1}\right) 2 I$ and similarly $S_{n} S_{n-1}=-S_{n-1} S_{n}+\left(\mathbf{S}_{n} \cdot \mathbf{S}_{n-1}\right) 2 I$ in the computation. Thus we see that, if we set $K_{n}=i \frac{-z^{3}+z+z^{-1}-z^{-3}}{4}\left(\mathbf{S}_{n} \cdot \mathbf{S}_{n-1}-\mathbf{S}_{n} \cdot \mathbf{S}_{n+1}\right) S_{n}+$ $i \frac{-z^{3}+z-z^{-1}+z^{-3}}{4}\left(\mathbf{S}_{n} \cdot \mathbf{S}_{n-1}-\mathbf{S}_{n} \cdot \mathbf{S}_{n+1}\right) I$, Eq.(2) is equivalent to holding the following prescribed discrete curvature representation:

$$
F_{n}^{A}:=\dot{L}_{n}-M_{n+1} L_{n}+L_{n} M_{n}=K_{n}, \quad \forall n .
$$

Our aim in this section is to geometrically transform Eq.(2) (resp., the connection (3)) to a discrete nonlinear Schrödinger-like equation (resp., a new connection) by a discrete gauge sequence $\left\{G_{n}\right\}$. So the key point is to find such a $\left\{G_{n}\right\}$. For this purpose, for a matrix sequence $\left\{S_{n}\right\}$ solving Eq.(2), we shall find $\left\{G_{n}\right\}$ to satisfy

$$
\sigma_{3}=-G_{n} S_{n} G_{n}^{-1}, \quad G_{n+1}=\left(\begin{array}{cc}
1 & \bar{q}_{n} \\
-q_{n} & 1
\end{array}\right) G_{n} \text { for some (complex valued) } q_{n},
$$

where $\sigma_{3}=\left(\begin{array}{cc}1 & 0 \\ 0 & -1\end{array}\right)$ is the Pauli matrix. The first equation of (5) has a class of solutions of the form

$$
G_{n}=\frac{i}{\sqrt{2\left(1-s_{n}^{1}\right)}}\left(\sigma_{3}-S_{n}\right) \operatorname{diag}\left(F_{n}, \bar{F}_{n}\right),
$$

where $\left\{F_{n}\right\}$ is free and to be specified latter. It is a straightforward verification that, in order for (6) to fulfill the second equation of (5) for some complex sequence $\left\{q_{n}\right\}, F_{n}$ is forced to satisfy the following iterated relation

$$
\left(1-s_{n}^{1}\right)\left(1-s_{n+1}^{1}\right) \overline{F_{n+1} F_{n}^{-1}}+\left(s_{n}^{2}-i s_{n}^{3}\right)\left(s_{n+1}^{2}+i s_{n+1}^{3}\right) F_{n+1} F_{n}^{-1}=2 \sqrt{\left(1-s_{n}^{1}\right)\left(1-s_{n+1}^{1}\right)}, \quad \forall n .
$$

So we may determine $F_{n}$ progressively by this relation in $n$ and hence have proved the existence of a desired gauge sequence $\left\{G_{n}\right\}$.

For the connection (3), we use $\left\{G_{n}\right\}$ to define a new connection $\left\{A_{n}^{G}=\left(L_{n}^{G}(t, z), M_{n}^{G}(t, z)\right)\right\}$ by $L_{n}^{G}(t, z)=G_{n+1} L_{n} G_{n}^{-1}=G_{n+1}\left(\frac{z+z^{-1}}{2} I+\frac{z-z^{-1}}{2} S_{n}\right) G_{n}^{-1}=\left(\begin{array}{cc}z^{-1} & z \bar{q}_{n} \\ -z^{-1} q_{n} & z\end{array}\right)$ and $M_{n}^{G}(t, z)=\dot{G}_{n} G_{n}^{-1}+G_{n} M_{n} G_{n}^{-1}=\dot{G}_{n} G_{n}^{-1}+i\left(\begin{array}{cc}\left(-1+z^{-2}\right) \frac{1}{1+\left|q_{n-1}\right|^{2}} & \left(1-z^{2}\right) \frac{\bar{q}_{n-1}}{1+\left|q_{n-1}\right|^{2}} \\ \left(1-z^{-2}\right) \frac{q_{n-1}}{1+\left|q_{n-1}\right|^{2}} & \left(1-z^{2}\right) \frac{1}{1+\left|q_{n-1}\right|^{2}}\end{array}\right)$. Here we have used the relations (5) in the computation. Since $L_{n}, M_{n}$ satisfy the prescribed discrete curvature representation (4), from Lemma 1 proved in [12] we know that $L_{n}^{G}, M_{n}^{G}$ should fulfill

$$
\dot{L}_{n}^{G}-M_{n+1}^{G} L_{n}^{G}+L_{n}^{G} M_{n}^{G}=G_{n+1} K_{n} G_{n}^{-1}, \quad \forall n .
$$


It is a direct computation that

$$
\begin{aligned}
& \text { l.h.s. of (7) }=\left(\begin{array}{cc}
1 & \bar{q}_{n} \\
-q_{n} & 1
\end{array}\right)\left[\left(\begin{array}{cc}
z^{-1} & 0 \\
0 & z
\end{array}\right) \dot{G}_{n} G_{n}^{-1}-\dot{G}_{n} G_{n}^{-1}\left(\begin{array}{cc}
z^{-1} & 0 \\
0 & z
\end{array}\right)\right] \\
& +i\left(\begin{array}{cc}
a_{n} & -\frac{\left(z^{-1}-z^{3}\right) \bar{q}_{n}}{1+\left|q_{n}\right|^{2}}+\frac{\left(z-z^{3}\right) \bar{q}_{n}+\left(z^{-1}-z\right) \bar{q}_{n-1}}{1+\left|q_{n-1}\right|^{2}} \\
-\frac{\left(z-z^{-3}\right) q_{n}}{1+\left|q_{n}\right|^{2}}+\frac{\left(z^{-1}-z^{-3}\right) q_{n}+\left(z-z^{-1}\right) q_{n-1}}{1+\left|q_{n-1}\right|^{2}} & b_{n}
\end{array}\right)
\end{aligned}
$$

where $a_{n}=\frac{\left(z^{-1}-z^{-3}\right)-\left(z-z^{-1}\right)\left|q_{n}\right|^{2}}{1+\left|q_{n}\right|^{2}}+\frac{\left(-z^{-1}+z^{-3}\right)+\left(z-z^{-1}\right) \bar{q}_{n} q_{n-1}}{1+\left|q_{n-1}\right|^{2}}$ and $b_{n}=-\frac{\left(z-z^{3}\right)+\left(z-z^{-1}\right)\left|q_{n}\right|^{2}}{1+\left|q_{n}\right|^{2}}+$ $\frac{\left(z-z^{3}\right)+\left(z-z^{-1}\right) q_{n} \bar{q}_{n-1}}{1+\left|q_{n-1}\right|^{2}}$ and

$$
\text { r.h.s. of (17) }=i\left(\frac{1}{1+\left|q_{n}\right|^{2}}-\frac{1}{1+\left|q_{n-1}\right|^{2}}\right)\left(\begin{array}{cc}
z^{-1}-z^{-3} & \left(-z+z^{3}\right) \bar{q}_{n} \\
\left(-z^{-1}+z^{-3}\right) q_{n} & -z+z^{3}
\end{array}\right) \text {. }
$$

Here we have used the relation (5) and some identities displayed in the appendix of [12]), e.g., $\mathbf{S}_{n} \cdot \mathbf{S}_{n+1}=\frac{1-\left|q_{n}\right|^{2}}{1+\left|q_{n}\right|^{2}}$ and $\mathbf{S}_{n} \cdot \mathbf{S}_{n-1}=\frac{1-\left|q_{n-1}\right|^{2}}{1+\left|q_{n-1}\right|^{2}}$, in the above computations. Thus, by substituting (8) and (9) into (7), the equation of the off-diagonal part of (7) leads to

$$
\dot{G}_{n} G_{n}^{-1}=\left(\begin{array}{cc}
* & i \frac{\bar{q}_{n}}{1+\left|q_{n}\right|^{2}}-i \frac{\bar{q}_{n-1}}{1+\left|q_{n-1}\right|^{2}} \\
i \frac{q_{n}}{1+\left|q_{n}\right|^{2}}-i \frac{q_{n-1}}{1+\left|q_{n-1}\right|^{2}} & *
\end{array}\right),
$$

where $*$ are some expressions which cannot be carried out at this moment. On the other hand, at the same time we also have

$$
\begin{aligned}
& \text { l.h.s. of (7) }=\left(\begin{array}{cc}
0 & \dot{\bar{q}}_{n} z \\
-\dot{q}_{n} z^{-1} & 0
\end{array}\right)+\left(\begin{array}{cc}
z^{-1} & \bar{q}_{n} z \\
-q_{n} z^{-1} & z
\end{array}\right) \dot{G}_{n} G_{n}^{-1} \\
& -\dot{G}_{n+1} G_{n+1}^{-1}\left(\begin{array}{cc}
z^{-1} & \bar{q}_{n} z \\
-q_{n} z^{-1} & z
\end{array}\right) \\
& +i\left(\begin{array}{c}
a_{n} \\
-\frac{\left(z-z^{-3}\right) q_{n}}{1+\left|q_{n}\right|^{2}}+\frac{\left(z^{-1}-z^{-3}\right) q_{n}+\left(z-z^{-1}\right) q_{n-1}}{1+\left|q_{n-1}\right|^{2}}
\end{array}\right)(11)
\end{aligned}
$$

By substituting (11) and (9) into the equation (7) and combining with (10), the diagonal part in this time implies

$$
\dot{G}_{n} G_{n}^{-1}=\left(\begin{array}{cc}
\alpha_{n} & i \frac{\bar{q}_{n}}{1+\left|q_{n}\right|^{2}}-i \frac{\bar{q}_{n-1}}{1+\left|q_{n-1}\right|^{2}} \\
i \frac{q_{n}}{1+\left|q_{n}\right|^{2}}-i \frac{q_{n-1}}{1+\left|q_{n-1}\right|^{2}} & \sigma_{n}
\end{array}\right)
$$

where $\alpha_{n}$ and $\sigma_{n}$ satisfy

$$
\left\{\begin{array}{l}
\alpha_{n}-\alpha_{n+1}=i\left(\frac{\bar{q}_{n} q_{n-1}}{1+\left|q_{n-1}\right|^{2}}-\frac{\bar{q}_{n+1} q_{n}}{1+\left|q_{n+1}\right|^{2}}\right) \\
\sigma_{n}-\sigma_{n+1}=i\left(\frac{q_{n+1} \bar{q}_{n}}{1+\left|q_{n+1}\right|^{2}}-\frac{q_{n} \bar{q}_{n-1}}{1+\left|q_{n-1}\right|^{2}}\right) .
\end{array}\right.
$$

Obviously, the second equation of (13) has a solution $\sigma_{n}=\bar{\alpha}_{n}$. By substituting (12) and (9) into (7), the off-diagonal part leads to $\left\{q_{n}\right\}$ satisfying the following discrete nonlinear Schrödinger-like equation

$$
\dot{q}_{n}+i \frac{q_{n+1}}{1+\left|q_{n+1}\right|^{2}}-2 i \frac{q_{n}}{1+\left|q_{n}\right|^{2}}+i \frac{q_{n-1}}{1+\left|q_{n-1}\right|^{2}}+\left(\alpha_{n}-\bar{\alpha}_{n+1}\right) q_{n}=0,
$$


where $\left\{\alpha_{n}\right\}$ solves the first equation of (13). This shows that the DHF (11) is the (discrete) gauge equivalent to the discrete nonlinear Schrödinger-like equation (14).

We would point out that Eq.(14) sets $\left\{A_{n}^{G}=\left(L_{n}^{G}(t, z), M_{n}^{G}(t, z)\right)\right\}$ as its discrete connection and has prescribed discrete curvature representation: $F_{n}^{G}=K_{n}^{G}$, where $K_{n}^{G}$ is given by right-hand-side of (9). Notice that the expression $K_{n}^{G}$ is to be zero at $z=1$, it can also be proved conversely that the discrete nonlinear Schrödinger-like equation (14) is (discrete) gauge equivalent to the DHF (10) by the gauge sequence $\left\{G_{n}\right\}$ satisfying $G_{n+1}=L_{n}^{G}(t, 1) G_{n}, \dot{G}_{n}=M^{G}(t, 1) G_{n}, \forall n$ (the existence of such a sequence $\left\{G_{n}\right\}$ is because of the integrability of $\left\{A_{n}^{G}\right\}$, or in other words its zero curvature representation, at $z=1$ ). The details are omitted here and one may refer to [12] for a reference.

$\left\{\alpha_{n}\right\}$ appeared in Eq.(14) can be solved out from the first equation of (13) as follows in different two approaches. One is obtained by iterating from initial data $q_{0}, q_{-1}$ and $\alpha_{0}$ :

$$
\alpha_{n}= \begin{cases}i \frac{\bar{q}_{n} q_{n-1}}{1+\left|q_{n}\right|^{2}}+i \sum_{k=1}^{n}\left(\frac{1}{1+\left|q_{k-1}\right|^{2}}-\frac{1}{1+\left|q_{k-2}\right|^{2}}\right) \bar{q}_{k-1} q_{k-2}-i \frac{\bar{q}_{0} q_{-1}}{1+\left|q_{-1}\right|^{2}}+\alpha_{0}, & n>0 \\ \alpha_{0}, & n=0 \\ i \frac{\bar{q}_{n} q_{n-1}}{1+\left|q_{n}\right|^{2}}+i \sum_{k=n}^{-1}\left(\frac{1}{1+\left|q_{k}\right|^{2}}-\frac{1}{1+\left|q_{k+1}\right|^{2}}\right) \bar{q}_{k+1} q_{k}-i \frac{\bar{q}_{0} q_{-1}}{1+\left|q_{0}\right|^{2}}+\alpha_{0}, & n<0 .\end{cases}
$$

The other is obtained by iterating from the boundary data at positive infinity:

$$
\alpha_{n}=i \sum_{k=n}^{+\infty}\left(\frac{1}{1+\left|q_{k}\right|^{2}}-\frac{1}{1+\left|q_{k+1}\right|^{2}}\right) \bar{q}_{k+1} q_{k}+i \frac{\bar{q}_{n} q_{n-1}}{1+\left|q_{n-1}\right|^{2}} \quad \forall n .
$$

This reflects that the property of the nearest neighbor exchange interaction for Eq.(11) collapses for its gauged equivalent equation (14), since the $n$-th chain's exchange interaction in Eq.(14) relates to chains from -1-st chain to $(n \pm 1)$-th chain or all the chains with label $\geqslant n-1$. Though this implies that the dynamics of Eq.(14) and hence the original Eq.(11) will be complicated, the exposition of the approximate linear stability in the next section for the stationary version of Eq.(14) shows in interesting fact: the $n$-th chain term is related to its nearest neighbor exchange interaction in the stability analysis. This reflects that Eq.(14) does have the property of the nearest neighbor exchange interaction in some sense. We note that $([4,7,12])$ the discrete nonlinear Schrodinger equations without nonconstant denominator terms (e.g. the AL equation: $i \dot{q}_{n}+\left(q_{n+1}+q_{n-1}-2 q_{n}\right)+\left|q_{n}\right|^{2}\left(q_{n+1}+q_{n-1}\right)=0$ ) are gauge equivalent to the (modified) discrete Heisenberg spin chain models with nonconstant denominator terms (e.g., the Ishimori equation: $\dot{S}_{n}=2 \frac{S_{n+1} \times S_{n}}{1+\mathbf{S}_{n+1} \cdot \mathbf{S}_{n}}+2 \frac{\left.S_{n} \times S_{n-1}\right]}{1+\mathbf{S}_{n} \cdot \mathbf{S}_{n-1}}$ ). And, meanwhile, the above exposition indicates that the discrete Heisenberg spin chain model (11) without nonconstant denominator terms is gauge equivalent to the discrete nonlinear Schrodinger-type equation (14) with nonconstant denominator terms. This is an interesting duality phenomenon between discrete nonlinear Schrodinger-type equations and discrete Heisenberg spin chain models.

Before we use (14) to study the transmission problem of the DHF, let's give a general description of constructing solutions to the DHF (1), or equivalently (2), from those to 
the discrete nonlinear Schrödinger-like equation (14). For a solution $\left\{q_{n}\right\}$ to Eq.(14), let $G_{0}$ be a fundamental solution to

$$
\dot{G}_{0}=M_{0}(t, 1) G_{0}=i\left(\begin{array}{cc}
\alpha_{0} & \frac{\bar{q}_{0}}{1+\left|q_{0}\right|^{2}}-\frac{\bar{q}_{-1}}{1+\left|q_{-1}\right|^{2}} \\
\frac{q_{0}}{1+\left|q_{0}\right|^{2}}-\frac{q_{-1}}{1+\left|q_{-1}\right|^{2}} & \bar{\alpha}_{0}
\end{array}\right) G_{0} .
$$

It is a direct verification that, by successive iteration,

$$
G_{n}=\left\{\begin{array}{c}
L_{n-1}^{G}(t, 1) G_{n-1}, \quad n>0 \\
\left.L_{n+1}^{G}(t, 1)\right)^{-1} G_{n+1}, \quad n<0
\end{array}= \begin{cases}L_{n-1}^{G}(t, 1) \cdots L_{0}^{G}(t, 1) G_{0}, & n>0 \\
\left(L_{n+1}^{G}(t, 1) \cdots L_{0}^{G}(t, 1)\right)^{-1} G_{0}, & n<0\end{cases}\right.
$$

solves $G_{n+1}=L_{n}^{G}(t, 1) G_{n}, \dot{G}_{n}=M^{G}(t, 1) G_{n}, \forall n$. Therefore $\left\{S_{n}=-G_{n}^{-1} \sigma_{3} G_{n}\right\}$ is a solution to the DHF (2) which corresponds to $\left\{q_{n}\right\}$ under the discrete gauge transformation.

\section{$\S 3$ Applications}

In this section, we shall use the gauged equivalent equation (14) to study dynamical properties of the discrete Heisenberg ferromagnetic spin chain (11). We mainly focus on exploring the transmission property of the DHF (11) and its related linear stability analysis for the stationary version of the equation (14).

\section{A Transmission properties}

In this subsection, we study as a physical application whether the wave transmission property $([13,16])$ of the discrete nonlinear Schrödinger-like equation (14) and transfer it to that of the DHF (11) under the action of discrete gauge transformations. In order to do these, let's consider the following recurrence equation originated from the discrete nonlinear Schrödinger-like equation (14) by setting $q_{n}(t)=\varphi_{n} \exp (-i E t)$ :

$$
-E \varphi_{n}+\frac{\varphi_{n+1}}{1+\left|\varphi_{n+1}\right|^{2}}-2 \frac{\varphi_{n}}{1+\left|\varphi_{n}\right|^{2}}+\frac{\varphi_{n-1}}{1+\left|\varphi_{n-1}\right|^{2}}-i\left(\alpha_{n}-\bar{\alpha}_{n+1}\right) \varphi_{n}=0,
$$

where $\varphi_{n}$ is a complex amplitude independent of the time variable $t, E$ is a real parameter and $\alpha_{n}$ independent of the time variable $t$ solves the first equation of (13), which reads from (16),

$$
\alpha_{n}=i \sum_{k=n}^{+\infty}\left(\frac{1}{1+\left|\varphi_{k}\right|^{2}}-\frac{1}{1+\left|\varphi_{k+1}\right|^{2}}\right) \bar{\varphi}_{k+1} \varphi_{k}+i \frac{\bar{\varphi}_{n} \varphi_{n-1}}{1+\left|\varphi_{n-1}\right|^{2}} \quad \forall n .
$$

We thus study the transmission problem of Eq.(19) via a similar numerical way of the AL-DNLS lattice chain displayed in [13, 16] (please refer to their papers for details). First we note that Eq.(19) has a stationary solution as follows:

$$
\varphi_{n}=T e^{i \kappa n}, \quad n \in \mathbf{Z}
$$


where the real parameter $E$ satisfies the consistent condition

$$
2 \cos \kappa=\frac{2+E\left(1+T^{2}\right)}{1+T^{2}} .
$$

We now consider the problem: A finite nonlinear segment $0 \leqslant n \leqslant N-1$ of length $N$ in the nonnegative stationary regime is embedded in a nonlinear chain $\left\{\varphi_{n}\right\}_{n \in \mathbf{Z}}$ satisfying (19) with

$$
\varphi_{n}=\left\{\begin{array}{lc}
R_{0} e^{i \kappa}+R e^{-i \kappa}, & n=0 \\
T e^{i \kappa n}, & n \geqslant N,
\end{array}\right.
$$

where (22) is fulfilled. This can be regarded as that an incident plane wave $R_{0} e^{i \kappa}$ on the left $(n=0)$ induces a reflected plane wave $R e^{-i \kappa}$ on the left and a transmitted plane wave $T e^{i \kappa n}$ on the right $(n \geqslant N)$. $R_{0}$ is called the amplitude of the incoming wave, $R$ the amplitude of the reflected waves and $T$ the transmitted amplitude at the right end of the nonlinear chain; $\kappa$ is called the out-coming wave number; $\left|R_{0}\right|^{2}$ and $|T|^{2}$ are also called the in-coming and the transmitted intensity respectively. The medium is completely nonlinear, thus the transmission coefficient $T$ as a function of $R_{0}$ may not uniquely determined. If this is true, according to the sense made in [17, 13], it is called bistability. For the forward transmission problem of the AL-DNLS lattice chain, there occurs exactly the bistability phenomenon ([13, 16]), which leads Delyon et al [13] to consider the backward transmitted problem for the DNLS. They proved that the pair $(\kappa,|T|)$ initializes the incident intensity $\left|R_{0}\right|$ completely and then displayed the transmission behavior of the DNLS in the $(\kappa,|T|)$ plane.

Now we consider the similar backward transmission problem for solutions with the type (23) to the present equation (19). Following Wan and Soukoulis [15], we use polar coordinates for $\varphi_{n}$, that is $\varphi_{n}=R_{n} e^{i \theta_{n}}$. For a given pair $(\kappa,|T|)$ (without loss of the generality, we may assume $T>0$ in this paper), we try to iterate Eq.(19) from $n=N$ to $-\infty$ successively and to determine the amplitudes $\left(R_{N-1}, \cdots, R_{0}\right)$ and phases $\left(\theta_{N-1}, \cdots, \theta_{0}\right)$. The existence of solutions with the type (23) to Eq.(19) will be shown below in the algorithms. In order to further support the existence of such solutions, an approximate linear stability analysis around the the stationary solution (21) will be given in the next section, which further reveals the linear stability behaviors of the equation (19). In this iterating process, we find that there are two choices in determining the amplitudes $\left(R_{N-1}, \cdots, R_{0}\right)$ in each step. This implies that, not like the transmission problem of the AL-DNLS, the pair $(\kappa, T)$ does not initialize the incident intensity $\left|R_{0}\right|$ completely, or in other words, the backward transmission problem for the equation (19) with solutions of type (23) is still bistable.

We first introduce a definite algorithm such that the pair $(\kappa, T)$ initializes the amplitudes $\left(R_{N-1}, \cdots, R_{0}\right)$ and phases $\left(\theta_{N-1}, \cdots, \theta_{1}\right)$ completely. In fact, such an algorithm is designed as follows. From $\varphi_{n}=T e^{i \kappa n}, n \geqslant N$ we get $\alpha_{n}=i \frac{\bar{\varphi}_{n} \varphi_{n-1}}{1+\left|\varphi_{n-1}\right|^{2}}, n \geqslant N$ from 
(20) and the equation (19) for $n \geqslant N+1$ is now equivalent to (22). The equation (19) for $n=N$ is rewritten as

$$
\left\{\begin{array}{l}
T \cos (\kappa)+\frac{1+T^{2}}{1+R_{N-1}^{2}} R_{N-1} \cos \left(\Delta \theta_{N}\right)=\frac{2+E\left(1+T^{2}\right)}{1+T^{2}} T \\
T \sin (\kappa)-\frac{1+T^{2}}{1+R_{N-1}^{2}} R_{N-1} \sin \left(\Delta \theta_{N}\right)=0
\end{array}\right.
$$

where $\Delta \theta_{n}=\theta_{n}-\theta_{n-1}$ (we remark that $\left.\theta_{N}=\kappa N\right)$, which leads $R_{N-1}$ to satisfying $T=\frac{1+T^{2}}{1+R_{N-1}^{2}} R_{N-1}$ and $\theta_{N-1}=\kappa(N-1)$. Since the quadratic equation $T=\frac{1+T^{2}}{1+x^{2}} x$ in $x$ just has two self-reciprocal solutions: $x=T$ and $x=\frac{1}{T}$, we then determine $R_{N-1}$ according to the rule: to choose $R_{N-1}>1$ when $0<T<1$ and $R_{N-1}<1$ when $T>1$ in order to avoid getting the stationary solution (21). Next, from (20) we see that, for $n<N, \alpha_{n}=i \sum_{k=n+1}^{N}\left(\frac{1}{1+\left|\varphi_{k-1}\right|^{2}}-\frac{1}{1+\left|\varphi_{k}\right|^{2}}\right) \bar{\varphi}_{k} \varphi_{k-1}+i \frac{\bar{\varphi}_{n} \varphi_{n-1}}{1+\left|\varphi_{n-1}\right|^{2}}$. Then the equation (19) for $n<N$ is is equivalent to

$$
\left\{\begin{aligned}
\frac{R_{n-1}}{1+R_{n-1}^{2}} \cos \left(\Delta \theta_{n}\right)= & \frac{R_{n}}{1+R_{n}^{2}}\left\{\frac{E\left(1+R_{n}^{2}\right)+2}{1+R_{n}^{2}}-\frac{R_{n+1}\left(\frac{1}{R_{n}}-R_{n}^{3}+2 R_{n}+2 R_{n} R_{n+1}^{2}\right)}{\left(1+R_{n}^{2}\right)\left(1+R_{n+1}^{2}\right)} \cos \left(\Delta \theta_{n+1}\right)\right. \\
& \left.-2 \sum_{k=n+1}^{N-1}\left(\frac{1}{1+R_{k}^{2}}-\frac{1}{1+R_{k+1}^{2}}\right) R_{k} R_{k+1} \cos \left(\Delta \theta_{k+1}\right)\right\} \\
\frac{R_{n-1}}{1+R_{n-1}^{2}} \sin \left(\Delta \theta_{n}\right)= & \frac{R_{n+1}}{1+R_{n+1}^{2}} \cos \left(\Delta \theta_{n+1}\right) .
\end{aligned}\right.
$$

The above exposition indicates that $R_{n-1}$ satisfies

$$
\frac{R_{n-1}}{1+R_{n-1}^{2}}=\sqrt{(\text { r.h.d of the first eq.(24) })^{2}+(\text { r.h.d of the second eq.(24) })^{2}}
$$

and thus also has two self-reciprocal solutions. Our rule is that in the second step and the following steps, we always choose the root with $R_{n}>1$ for the quadratic equations. According to this algorithm, we may uniquely determine the amplitudes and phases from $n=N-1$ to $-\infty$ successively and especially the incident intensity $\left|R_{0}\right|^{2}$. This procedure also indicates the existence of solutions of type (23) to the stationary equation (19).

If the resulting incoming wave intensity $\left|R_{0}\right|^{2}$ is of the same order of $\frac{1}{|T|^{2}}$ (the reciprocal transmitted intensity $|T|^{2}$ ) independent of $N$ when $0<T<1$, or is of the same order of $|T|^{2}$ when $T>1$, we say that the nonlinear chain with wave number $k$ and outgoing intensity $|T|^{2}$ is to be transmitting. If $R_{0}$ appears to be a rapidly increasing function of $N$, we say that this nonlinear chain is to be non-transmitting. Figs.1(a) and (b) below display the transmission behaviors in the $(k, T)$ parameter plane with the chain length $N=110$ and 150, respectively, representing region of transmitting (white) and nontransmitting (black) behaviors. In particular, Fig.1 (a) and (b) numerically show the whole-scope of the transmitting behaviors for $\kappa \in(1,3.5)$ and $T \in(0.5,2)$. The nontransmitting regions form some strange patterns. The enlargement of one of these patterns, $(\kappa, T) \in(1.3,1.7) \times(1,1.4)$, is further shown by Fig.1(c). It is seen from the figure that the twisting boundaries between the transmitting region and the non-transmitting region 
in the parameters plane always display spiral, cantor-like, and fractal structure, which is very analogous to the structure of the standard chaotic attractor. Furthermore, from the Fig.1 (a) and (b), we see that the transmitting behaviors only depend seriously on the parameters $\kappa$ and $T$ but do not depend sensitively on the chain length $N$.

Figure 1 around here. Please find it at the end of the paper.

Besides the definite algorithm, we come to consider a stochastic algorithm. In such a stochastic algorithm, the determination of the bi-valued $R_{N-1}$ should be the same as that in the definite algorithm in order to avoid getting a stationary solution; however, the latter determinations of $R_{n}(0 \leqslant n \leqslant N-2)$ in each step are based on the generation of a random number $\xi$, i.e., $R_{i}=R_{-}$if $\xi \geqslant c ; R_{i}=R_{+}$otherwise. Naturally, our first choice of the generation of the random number $\xi$ is the normal distribution and the value $c$ is taken as zero, the expectation of this distribution. It could be seen from Fig.2 (a) that nontransmitting regions (black) periodically appear with respect to $\kappa$, here the chain length is still to be $N=150$. Also, this kind of phenomena could be somewhat found in Fig.1 (a) and (b). Secondly, a Poisson distribution with an expectation 5 is adopted to produce the random number $\xi$ and $c$ is taken as 5 . In this case, a similar periodic non-transmitting behavior is displayed in Fig2.(b). Actually, thousands of numerical simulations verify a fact that no matter what kind of stochastic distribution and chain length are taken, the non-transmitting behavior is so prevalent around the values $\kappa_{k} \approx 1.57+\frac{k \pi}{2}(k=0,1, \cdots)$. This periodic phenomena could be regarded as an invariant non-transmitting behavior for Eq.(19).

Figure 2 around here. Please find it at the end of the paper.

The transmission problem associated with the discrete Heisenberg ferromagnetic spin chain (11) (or equivalently (2)) is now proposed as follows. There is a semi-infinite nonlinear matrix wave chain $\left\{S_{n}=-G_{n}^{-1} \sigma_{3} G_{n}\right\}$ embedded in a nonlinear chain of the DHF (2), where $G_{n}$ looks like (from (18)):

$$
G_{n}=\left(\begin{array}{cc}
1 & \bar{q}_{n-1} \\
-q_{n-1} & 1
\end{array}\right) \cdots\left(\begin{array}{cc}
1 & \bar{q}_{0} \\
-q_{0} & 1
\end{array}\right) G_{0}, \quad n \geqslant 1
$$

with $G_{0}$ satisfying (17) and $q_{n}=\varphi_{n} e^{-i E t}$, where $\varphi_{n}$ is independent of $t$ and given by (23). This nonlinear matrix wave chain is from the left towards the right, where they are scattered into reflected and transmitted parts. Similarly, $R_{0}, R$ present the amplitudes of the incoming and reflected waves and $T$ the transmitted amplitude at the right end of the nonlinear chain under consideration. $\left|R_{0}\right|^{2}$ and $|T|^{2}$ are also called the incoming wave intensity and the transmitted intensity of the nonlinear matrix chain respectively. As we have proved in the previous section that the DHF (1) (i.e. the DHF (2) ) is the discrete gauge equivalent to the discrete nonlinear Schrödinger-like equation (14) and verse visa, we see that the transmission properties of the equation (19) just displayed, i.e., the above definite algorithm and stochastic algorithms, are transferred completely to the transmission problem (25) of the DHF (2) under the action of discrete gauge transformations. 
Thus the nonlinear matrix wave chain (25) of the DHF (2) has the same transmission behaviors as those of nonlinear chain (23) of Eq.(19). Fig.1(a),(b) and (c) are completely suitable in displaying the transmission behaviors of the finite nonlinear matrix chain of the DHF (2) in the parameters $(k, T)$ plane with chain length $N=110$ and 150 under the definite algorithm and so do Fig.2(a) and (b) under stochastic algorithms. Furthermore, from the Fig.1 (a) and (b), we see that the transmitting behaviors for solutions $\left\{S_{n}=-G_{n}^{-1} \sigma_{3} G_{n}\right\}$ with $\left\{G_{n}\right\}$ satisfying (25) to the DHF (11) only depend seriously on the parameters $\kappa$ and $T$ but do not depend sensitively on the chain length $N$. This shows that the important phenomenon of bistability occurs still in spin magnetic physics. The periodic phenomenon of the non-transmitting regions displayed in Fig.2 provides a new and interesting transmission property of the DHF (1).

\section{B Stability analysis}

In order to support the exposition in subsection A and show the existence of stable solutions to (19) around the stationary solution (21), we shall give an approximate linear stability analysis for Eq. (19). In what follows, we focus on the locally linear stability of the stationary solution (21) in the reverse iterative procedure through the linear variational method [19, 20]. For this purpose, we naturally import the formulas as follows:

$$
R_{n}=T+\tau_{n}, \quad \theta_{n}=n \kappa+\delta_{n}, \quad n=N, N-1, \cdots, 2,1,
$$

where quantities $\tau_{n}$ and $\delta_{n}$, respectively, are regarded as small perturbations of the modulus and argument of the stationary solution (21). Substitute the above formulas into Eq. (24) and then expand those nonlinear terms in the vicinity of $T$ and $n \kappa$. A tedious calculation thus gives the following equations at $O\left(\tau_{i}\right)$ and $O\left(\delta_{i}\right)(i=n-1, n, n+1)$ :

$$
\left\{\begin{aligned}
\frac{\left(1-T^{2}\right) \cos \kappa}{\left(1+T^{2}\right)^{2}} \tau_{n-1}= & \frac{1-T^{2}}{\left(1+T^{2}\right)^{2}}\left[E+\frac{2}{1+T^{2}}-\frac{\cos \kappa}{1+T^{2}}-\frac{T^{2} \cos \kappa}{1+T^{2}}\right] \tau_{n} \\
& +\frac{T}{1+T^{2}}\left[-\frac{4 T}{\left(1+T^{2}\right)^{2}}+\frac{\cos \kappa}{T\left(1+T^{2}\right)}-\frac{2 T\left(1-T^{2}\right) \cos \kappa}{\left(1+T^{2}\right)^{2}}\right] \tau_{n} \\
& +\frac{T}{1+T^{2}}\left[-\frac{\left(1-T^{2}\right) \cos \kappa}{T\left(1+T^{2}\right)^{2}}--\frac{2 T \cos \kappa}{1+T^{2}}+\frac{2 T\left(1-T^{2}\right) \cos \kappa}{\left(1+T^{2}\right)^{2}}\right] \tau_{n+1} \\
& +\frac{2 T^{4} \cos \kappa}{\left(1+T^{2}\right)^{2}} \sum_{k=n+1}^{N-1}\left(\tau_{k}-\tau_{k+1}\right), \\
\frac{\sin \kappa}{1+T^{2}}\left(\delta_{n}-\delta_{n-1}\right)= & -\frac{\sin \kappa}{1+T^{2}}\left(\delta_{n+1}-\delta_{n}\right), \\
\frac{\left(1-T^{2}\right) \sin \kappa}{\left(1+T^{2}\right)^{2}} \tau_{n-1}= & \frac{\left(1-T^{2}\right) \sin \kappa}{\left(1+T^{2}\right)^{2}} \tau_{n+1}, \\
\frac{T \cos \kappa}{1+T^{2}}\left(\delta_{n}-\delta_{n-1}\right)= & \frac{T \cos \kappa}{1+T^{2}}\left(\delta_{n+1}-\delta_{n}\right),
\end{aligned}\right.
$$

where $n=N, N-1, \cdots, 2,1$. Clearly, combining the second and the fourth equations in (26) leads to

$$
\delta_{n+1}-\delta_{n}=0,
$$

which shows an identity of the series $\left\{\delta_{n}\right\}$ at $O\left(\delta_{n}\right)$. Hence, the linear stability of the argument of the stationary solution in the reverse iteration procedure belongs to the stable case. However, the nonlinear stability of this argument becomes a critical case, depending 
on the further calculation at $O\left(\delta_{i}^{2}\right)$. Due to the tediousness of notations, we omit the calculation here. Analogously, combining the first and the third equations in (26) yields:

$$
\tau_{n-1}=\left[\frac{1+T^{4}}{1-T^{4}}-\frac{2 T^{2}}{\left(1-T^{4}\right) \cos \kappa}\right] \tau_{n}-\frac{2 T^{4}}{1-T^{4}} \tau_{N} .
$$

Hence, it follows that for $n=N, \cdots, 1$,

$$
\begin{aligned}
\tau_{n-1}= & \left\{\left[\frac{1+T^{4}}{1-T^{4}}-\frac{2 T^{2}}{\left(1-T^{4}\right) \cos \kappa}\right]^{N-n+1}\right. \\
& \left.-\frac{2 T^{4}}{1-T^{4}} \sum_{j=0}^{N-n}\left[\frac{1+T^{4}}{1-T^{4}}-\frac{2 T^{2}}{\left(1-T^{4}\right) \cos \kappa}\right]^{j}\right\} \tau_{N} \triangleq \lambda_{N, n} \tau_{N} .
\end{aligned}
$$

Consequently, the linear stability of the modulus of the stationary solution is stable in the reverse iteration provided that $\lambda_{N, n}$ is uniformly bounded for all $N$ and $n$. More precisely, the reverse stability holds when the following inequalities are satisfied:

$$
\left|\frac{1+T^{4}}{1-T^{4}}-\frac{2 T^{2}}{\left(1-T^{4}\right) \cos \kappa}\right|<1
$$

In addition, notice that $\tau_{n}=\lambda_{n, 0}^{-1} \tau_{0}$. This implies that the linear stability of the normal iteration procedure is stable when $\lambda_{n, 0} \neq 0$ for all $n$. Thus, this linear stability is valid only provided

$$
\kappa \neq \frac{k \pi}{2}(k=0, \pm 1, \pm 2, \cdots)
$$

Obviously, this necessary stability condition is also included in the condition (27). As shown in Fig.3, parameters selected from the black regions violate the inequality (27). Those diamond-like unstable regions periodically appears in a period $2 \pi$ with respect to the parameter $\kappa$.

Figure 3 around here. Please find it at the end of the paper.

The relation of the linear stability analysis in this subsection and the transmission exposition in the subsection $\mathrm{A}$ is that the non-transmitting regions in Figure 1 or Figure 2 are always contained in the unstable regions in Figure 3 (b). This also indicates that the transmission exposition for solutions with the type (23) to Eq.(19) is a more accurate description of the linear stability analysis for Eq.(19), which exhibits some chaotic dynamics of the Eq.(14) and hence those of the DHF (1).

\section{$\S 4$ Conclusion}

We present a mechanism for displaying the transmission property of the discrete Heisenberg ferromagnetic spin chain via a geometric approach. More precisely, we first transform the DHF (11) into the discrete nonlinear Schrödinger-like equation (14) with the aid of the (discrete) Yang-Mills theory. Then we use the stationary version of the discrete nonlinear Schrödinger-like equation (14) to study the transmission problem of the DHF (11). In this 
procedure, we show that the determination of transmitting coefficients in the backward transmission problem is always bistable. Thus a definite algorithm and general stochastic algorithms are presented. The corresponding transmitting behaviors in the parameters $(\kappa, T)$ plane are shown by Figure 1 and 2. A new invariant periodic phenomenon of the non-transmitting behavior for the DHF, with a large probability, is revealed by an adoption of various stochastic algorithms. By the way, an approximate linear stability analysis for the stationary version of Eq.(19) is also given for supporting the discussion of the transmission expositions. We remark that though the gauged equivalent equation (14) with (13) looks much complicated and we cannot give a direct physical application at present stage from it, it is an effective way to utilize it to study some dynamical properties of the DHF (1). We believe that the discrete nonlinear Schrödinger-like equation (14) can help us to reveal much more and deeper quantum chaotic properties of the DHF (1).

\section{Acknowledgments}

This work was supported by the National Natural Science Foundation of China (Grant Nos. 10531090, 10501008) and STCSM.

\section{References}

[1] N. Papanicolaou, J. Phys. A: Math. Gen. 20 (1987) 3637.

[2] M. Laksmanan, Phys. Lett. A 61 (1977) 53.

[3] L.A. Takhtajan, Phys. Lett. A 64 (1977) 235.

[4] Y. Ishimori, J. Phys. Soc. Japan, 52 (1982) 3417.

[5] K. Porsezian, M. Daniel and M. Lakshmanan, J. Math. Phys. 33 (1992) 1907.

[6] M. Daniel and R. Amuda, Phys. Rev. B 53 (1996) R2930.

[7] Q. Ding, Phys. Lett. A 266 (2000), 146.

[8] H.J. Mikeska and M. Steiner, Adv. Phys. 44 (1991) 191.

[9] V.E. Zakharov and L.A. Takhtajan, Theor. Math. Phys. 38 (1979) 17.

[10] L.D. Faddeev and L.A. Takhtajan, Hamiltonian Methods in the Theorey of Solitons, SpringerVerlag, Berlin, Heideberg 1987.

[11] Q. Ding and Z. Zhu, J. Phys. Soc. Japan 72 No.1 (2003), 49.

[12] Q. Ding, J.Phys. A: Math. Theor. 40 (2007) 1991.

[13] F. Delyon, Y.E. Levy and B. Souillard, Phys. Rev. Lett. 57 (1986) 2010.

[14] B.M. Herbst, and M.J. Ablowitz, Phys. Rev. Lett. 62 (1989) 2065. 
[15] Yi Wan and C.M. Soukoulis, Phys. Rev. A 41,800 (1990)

[16] D. Hennig, N.G. Sun, H. Gabriel and G.P. Tsironis, Phys. Rev. E 52 (1995) 255.

[17] C. Flytzanis, in Nonlinear Phenomenon in Solids, edited by A.F. Vavrek (World Scientific, Singapore, 1985).

[18] P.G. Kevrekidis, K. Rasmussen and A.R. Bishop, Inter. J. Mod. Phys. B 15 (2001) 2833.

[19] C. Robinson, "Dynamical Systems: Stability, Symbolic Dynamics, and Chaos," 2nd Ed., CRC Press, 1998.

[20] R. Grimshaw, "Nonlinear Ordinary Differential Equations," Blackwell, Oxford, 1990. 


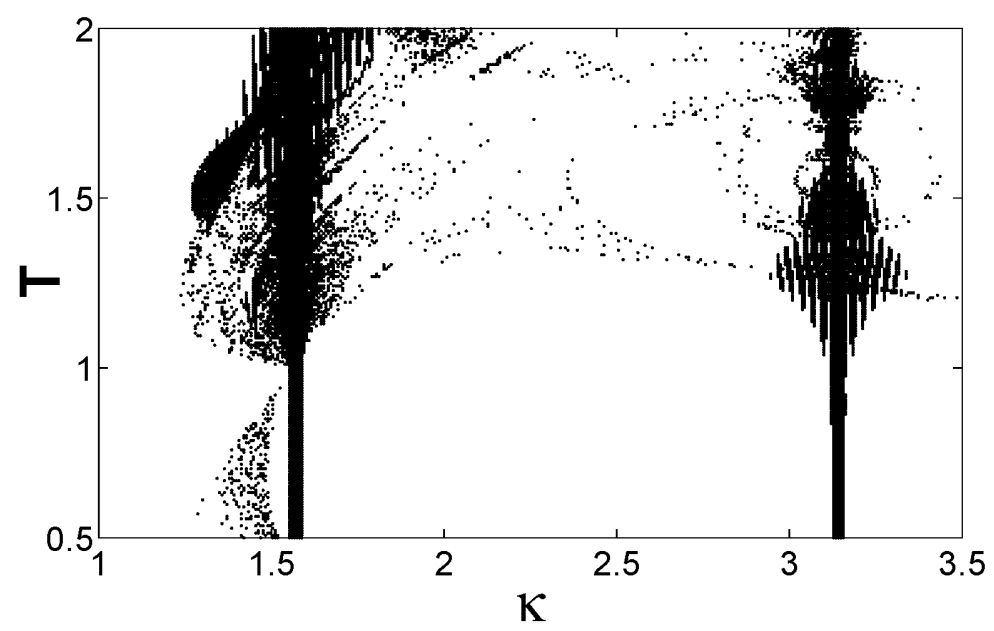

(a)

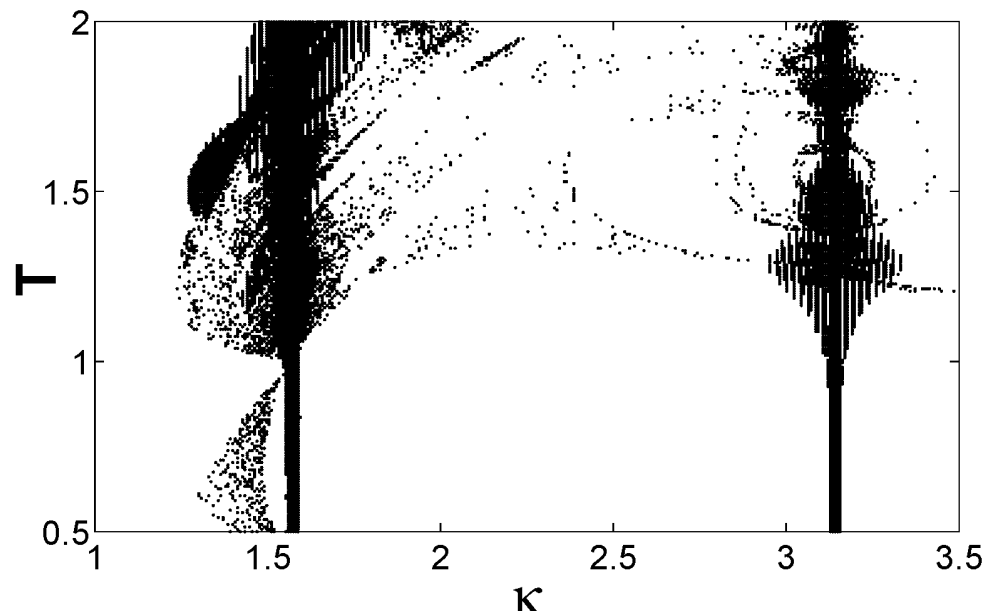

(b)

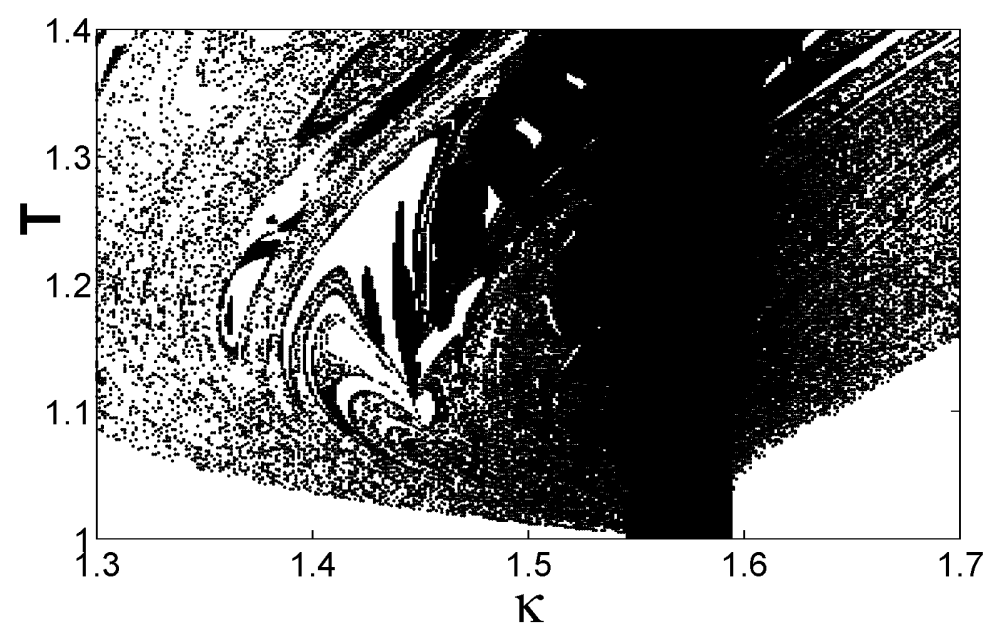

(c)

Fig. 1: The transmitting behavior in the $(\kappa, T)$ parameters plane $(\kappa, T) \in$ $(1,3.5) \times(0.5,2)$ (a) with $N=110$ and (b) with $N=150$, and in an enlarged region $(\kappa, T) \in(1.3,1.7) \times(1,1.4)(\mathrm{c})$ with $N=150$. The boundaries of transmitting (white) and non-transmitting (black) regions exhibit fractal-like structure. . 


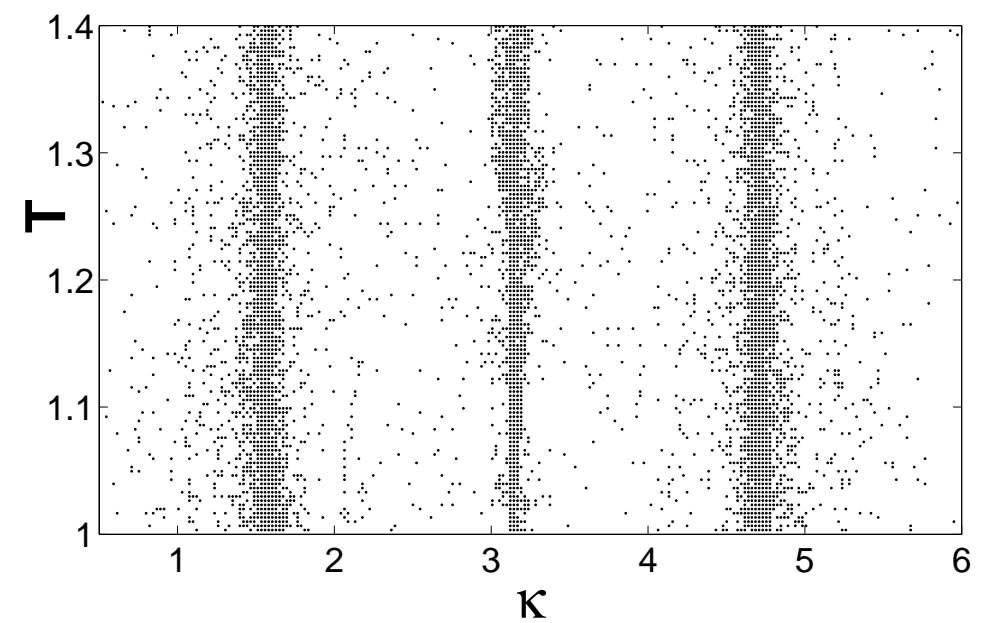

(a)

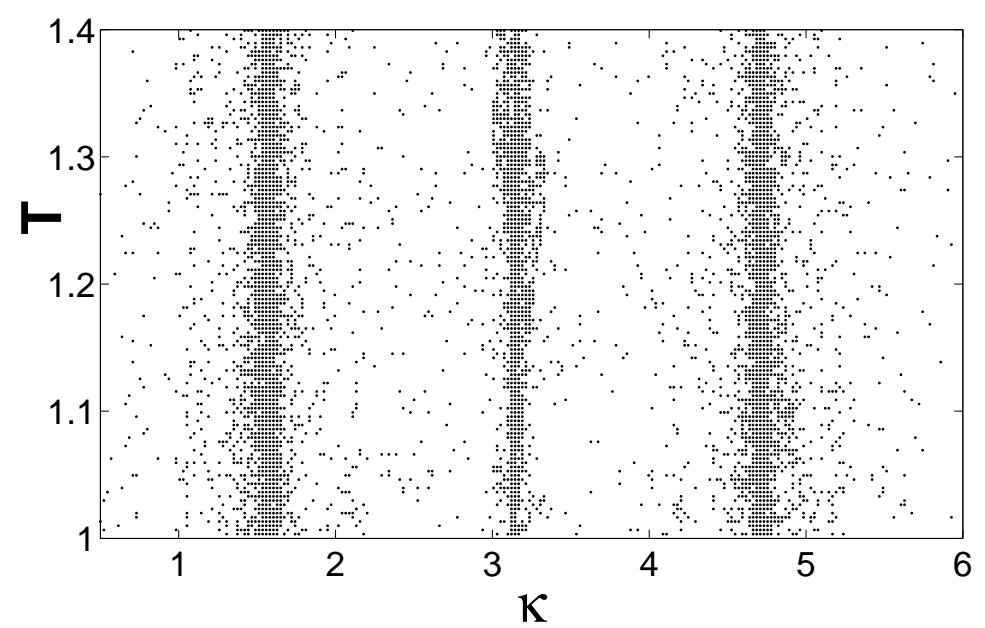

(b)

Fig.2. The periodic phenomenon of the non-transmitting behavior in $(\kappa, T)$ plane by adopting of the normal distribution (a) and a Poisson distribution (b). Here the chain length $N=150$. 


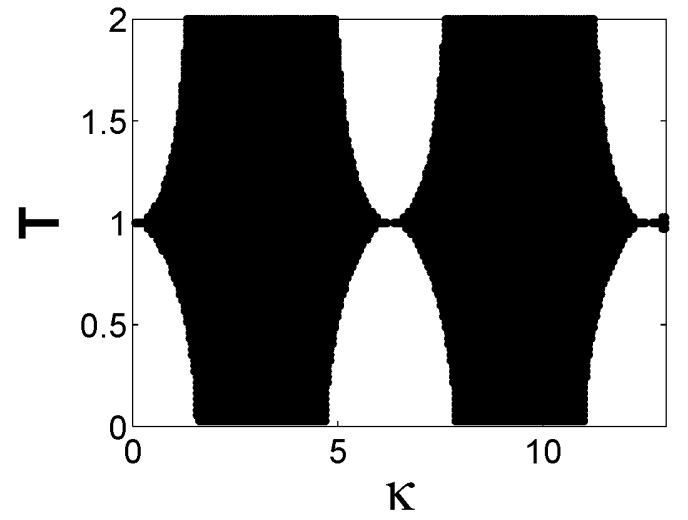

(a)

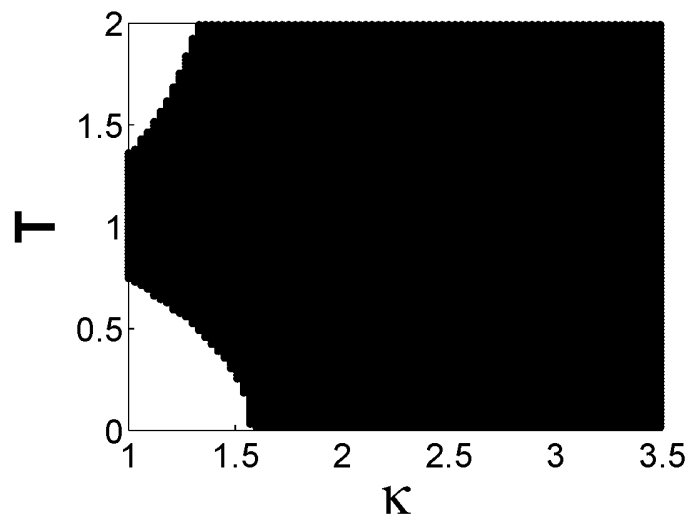

(b)

Fig.3. The linear stability parameters regions (white for stable region and black for unstable region) when $(\kappa, T)$ is in $(0,13) \times(0,2)(\mathrm{a})$ and in $(1,3.5) \times(0,2)(\mathrm{b})$, respectively. 